\title{
Acoustical Method and Device for Precipitation Enhancement Inside Natural Clouds
}

\author{
Tamara Tulaikova, Svetlana Amirova \\ Moscow Institute of Physics and Technology, Institute per.9, Moscow Region, Dolgoprudny, Russia
}

Email address:

amirova.svetlana@yahoo.com (S. Amirova), tulaikova@gmail.com (T. Tulaikova)

\section{To cite this article:}

Tamara Tulaikova, Svetlana Amirova. Acoustical Method and Device for Precipitation Enhancement Inside Natural Clouds. Science Discovery. Special Issue: New Technical Ideas for Climate Recovery. Vol. 3, No. 2-1, 2015, pp. 18-25. doi: 10.11648/j.sd.s.2015030201.13

\begin{abstract}
Acoustic method was analyzed to obtain fast droplets coalescence inside nature clouds up to rainy big sizes with gravity sedimentation. Droplets are triggered by sound waves which provide the same high speed for coalescence process near $300 \mathrm{~m} / \mathrm{s}$. Optimal acoustic generator is proposed, optimal regimes for cloud droplets have been found at low frequencies of $f \sim$ $100 \mathrm{~Hz}$ with low acoustical power. Aadditional acoustic possibilities for rain stimulations provide fast improvement for hot arid weather in local areas on tens-kilometer scales.
\end{abstract}

Keywords: Atmosphere, Precipitation Enhancement, Clouds, Acoustics

\section{Introduction}

The idea of weather modification by precipitation seeding was generated earlier by Vincent Schaefer, Irving Langmuir, and later Arnett S. Dennis [1-2]. The most popular methods used today for precipitation enhancement are the sprinkling of hygroscopic particles or a special solutions for 'warm' clouds and the introduction of glaciogenic substances into 'cold' clouds from an aircraft, see [3-7] and etc. From the analysis of the relationship between the state of equilibrium for the cloud condensation nuclei and the relative air humidity, the resulting preference of special soluble particles of substances was proven for the modification of warm or cold clouds, but further coalescence processes should be effectively continued with vibrations of the drop ensemble through the application of special acoustical power inside the clouds $[8,9]$. The acoustically influencing for mists and fogs was widely used early, see [10-13] and etc. The method of acoustic coalescence of aerosols in industry and for mists precipitation was developed earlier in the 1950-1960s. Unfortunately the industrial aerosols was the central purpose for the acoustical seeding in these years. There were many plant's units in these times in USA, Germany, Russia, and etc. for acoustical seeding of industry pollution, common practice show the efficiency up to $99 \%$. There were series of publications with theoretical models for the aerosol coagulation by acoustical influence. Some experiments were done with rains as a result in Russian Caucasus then high- power acoustical irradiation was located on mountain to affect to clouds directly [12]. However, this method was not applied later in practice for clouds due to weak technique, but another method using hygroscopic particles was simple during utilization in clouds.

At the same time, the air over heating became strong now with each next year due to effects of global warming at the planet [14-16]. Acoustic additional possibilities for stimulation rains serve for fast development of series of precipitation enhancement. The weather improvement could be realized in local area with 100-kilometers scale, but stimulated instability processes could have further distance/time development for precipitations. The careful study of the available literature of acoustical coagulation of aerosols shows that sound waves for utilization in clouds was not developed enough. Firstly, there were not enough connection of a complete theory with the experiments; this means that most experiments were completed without enough theoretical initiation or explanations; they were performed by different groups of scientists. The main objects for acoustic-based purification were industry aerosols, but no droplets in clouds that is larger one and with another nature. At last, acoustical experiments were only carried out near the ground, but it could be most effective to directly place the sound generator inside a cloud in the region with oversaturated water steam by using a modern aircraft (helicopters). The most interesting perspective for today is a joint utilization of both methods at the same time, which 
means that hygroscopic particles and acoustic influence would be directly applied inside one cloud area.

Physics show special rule and necessity for acoustics in clouds. Dynamics of different hygroscopic particles show that only first $15 \div 20$ seconds demonstrate very fast changes in the main cloud characteristics as the supersaturation and spectrum dispersion. This is accompanied by an increase of the radii inside small drops from $0.01 \div 0.1 \mu \mathrm{m}$ up to $1 \div 3$ $\mu \mathrm{m}$. It could be understood as initiation of water vapor condensation onto surface of hygroscopic particle from surrounding, but next stage is the evaporation from the host cloud droplets with further condensation to new particle, such reorganization need much time. So, after the first major variations of these parameters they increase with the same tendencies very slowly, so significant changes in spectrum of cloud and precipitation increase up to time $30-50$ minutes [6]. As a result, the hygroscopic particles prove to be a very effective reorganization only within smallest drops with water vapors in the first few seconds. Particle's method really shows later a slow cloud reorganization by hygroscopic mechanisms due an additional collisions necessity. The idea is that further motion of resulted drops could be triggered by sound waves in the form of vibrations to provide the coalescence to a drop size of more than $100 \mu \mathrm{m}$ and after this point gravity will predominate. The background is the facts that acoustics provides high mobility for droplets, because they become be involving into air vibrations inside sound waves. The best coalescence efficiency appropriates to large and intermediate-sized cloud droplets. The speed of acousticbased vibrations and other stimulated processes for drops should be effective in clouds due to high speed of acoustic waves $(\mathrm{C} \sim 340 \mathrm{~m} / \mathrm{sec})$ in comparison with typical atmosphere winds $5-10 \mathrm{~m} / \mathrm{sec}$.

At present time, the hygroscopic particles should be imported by the airplane into special lowest part of natural convective clouds usually, where rising air flows exist with high velocity and in power cloud. And besides, great number of such experiments with hygroscopic particles didn't brig any results, especially in wide seaside areas. On the other hand, an additional acoustical action can provide necessary air flow by variable acoustic energy at any place immediately. The acoustical stimulation will increase the areas in atmosphere for the possibility of application of this method, also the number of cases depending weather conditions for mentioned active action will be also increased with results as a stable rains.

\section{Sound Attenuation in Cloud Media}

The propagation, absorption and attenuation of sound in a humid atmosphere, in clouds and in turbulent media are important topics. The amplitude and air pressure of ideal sound decreases with distance $(x)$ in ideal media, as follows $P=P_{0} \exp (-\alpha x)$. The absorption coefficient $\alpha$ of sound in the medium is expressed in $\mathrm{m}^{-1}$. Attenuation $\beta$ may be expressed in $\sec ^{-1}$, then the value of $\alpha\left(\mathrm{m}^{-1}\right)$ must be multiplied by the speed of sound. For the absorption coefficient in the compressible fluid the next relation is valid for estimations:

$$
\begin{aligned}
& \alpha=\frac{b \cdot \omega^{2}}{2 \rho \cdot C_{a}^{3}}, \\
& b=\frac{4}{3} \eta+\frac{\gamma-1}{C_{p}} \cdot a_{T}+\eta^{\prime} \approx 2.5 \cdot \eta
\end{aligned}
$$

There $\omega$ is a sound circular frequency in air, $\eta$ is the dynamic viscosity, the $C_{p}$ heat capacity at constant pressure and the ratio of specific heat capacities is $\gamma$, the $a_{T}$ is a thermal conductivity $\left(\mathrm{m}^{-1}\right)$. Theory predicts the absorption maximum in the range of few tens of $\mathrm{Hz}$. At lower frequencies, the time for thermal relaxation is large so to establish equilibrium between droplets with steam near them. At higher frequencies, on the contrary, the processes of evaporation and condensation do not have enough time and state is classified as an adiabatically dry, the absorption coefficient is low. Measurements are presented at Table 1 for the more interesting range for our further consideration.

Table 1. The sound absorption in the water mist then water liquid content is $W=2 \mathrm{~g} / \mathrm{m}^{3}$.

\begin{tabular}{llllllll}
\hline $\mathrm{F}, \mathrm{Hz}$ & 27.5 & 58 & 112 & 150 & 200 & 350 & 500 \\
$\beta, \mathrm{dB} / \mathrm{s}$ & 4.8 & 3.5 & 2.8 & 3.6 & 6.7 & 7.2 & 5.0 \\
\hline
\end{tabular}

Measurements and calculations indicate the sound attenuation is small in dry air, $\alpha \sim 10^{-3} \div 10^{-2} \mathrm{~dB} / \mathrm{m}$ then the sound frequency is $f=3 \div 10 \mathrm{kHz}$. As the humidity increases up to $12 \div 20 \%$ the attenuation increases up to $0.17 \div 0.56$ $d B / m$. For higher frequencies the attenuation does not depend strongly on the humidity and give the values: $\alpha=0.15 \div 0.05$ $\mathrm{m}^{-1}$ then $f=20 \mathrm{kHz} ; \alpha=3 \div 5 \mathrm{~m}^{-1}$ then $f=50 \mathrm{kHz}$; and $\alpha=7$ $\div 10 \mathrm{~m}^{-1}$ then $f=100 \mathrm{kHz}$, there humidity interval is $40-$ $100 \%$. Further it is equal to $\alpha=80 \mathrm{~dB} / \mathrm{m}$ then $f=200 \mathrm{kHz}$ for dry and wet air no matter. Sound attenuation in the air/water mist was measured [12]. Using measured data at Tabl.1 we deduce that a low frequency will propagate $\sim 500$ meters in typical clouds prior to decreasing in energy by a factor of 2 .
For the distance $0.5 \mathrm{~km}$ in $100 \%$-wet air, the calculations indicate a $40 \%$ or $90 \%$ signal decrease at frequencies 112 or $1000 \mathrm{~Hz}$, respectively.

The $0.5 \mathrm{~km}$ is enough distance in cloud for initiation the reorganization for precipitation to begin. In practice, the most effective glaciogenic particles with diameters of $5-10 \mathrm{~mm}$ run the distance of $\sim 0.1 \mathrm{~km}$ prior to evaporation or sublimation, and provide enough cloud reorganization for precipitation. The effects of the partial dissipation of irradiated sound waves during their propagation in a turbulence medium have been considered in [17-19], and additional publications. Clouds with plenty or prolonged rainfall are identified as $N s, A s, C b$ and $C u$, they are more 
important for the active actions of precipitation enhancement. Autumn and spring clouds are mixed with water droplets, ice crystals and snowflakes. The authors of papers [20-21] studied the formation and shapes of cloud fractions using both experiments and theories, they gave convenient empirical formula that can be employed in order to determine the percentage of water vapor, liquid, and solid phases in clouds created by the adiabatic process. The formula contains the factor of atmosphere pollution that is equal to $0 \div 3$, in the typical temperature range and for cloud droplets with radii of $r>24 \mu \mathrm{m}$.

\section{New Model for Acoustic Wave Affecting to the Clouds Droplets}

The purposes of the analyses in this chapter is to calculate an amplitude, $L(r, \omega)$, during the vibrations of cloud droplets with typical sizes inside acoustic wave with no high optimal power. The optimal regimes below were analyzed and calculated for the ensemble of cloud droplets. The optimal frequency $(f)$ should be low enough with an appropriate decrease in the acoustical power, $Q$. To decrease the weight and size of the acoustic generator together with appropriate pump systems to place them to helicopter, minimization of sound power is desirable from a technical perspective. Since the time of unidirectional motion of the drop decreases with increasing frequency up to $1 \mathrm{kHz}$, too small droplet displacement at high frequency is natural. On the other hand, the frequency decrease $f<1 \mathrm{kHz}$ will increase the time for a one-direction drop displacement during the half period time for sound waves $0.5 / f>500 \mathrm{~ms}$. Typical clouds have droplet radii within the range from $r \approx 1-50 \mu \mathrm{m}$, and the objective for the acoustics is the additional motion of the droplet ensemble in receiving greater droplets with radii $r \geq 100 \mu \mathrm{m}$.

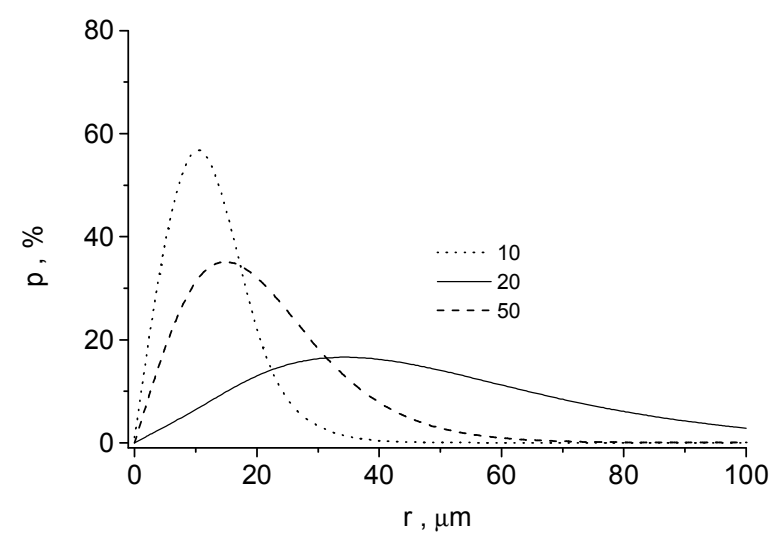

Figure 1. Calculated spectrum from cloud to rain then average droplet radius is $r_{0}=10,20$, or $50 \mu \mathrm{m}$.

First stages of rainy cloud formation corresponds to the ensemble with the average radius $r_{0}=3-5 \mu \mathrm{m}$. The increase in the average radius, $r_{0}$, with appropriate spectrum modifications up to flat curves match to rainy clouds.
Calculations according to approximation Masin-Hrgian formula (2) show the cloud spectrum evolution, presented at Figure 1:

$$
n(r)=a r^{2} \exp (-b r)
$$

Empirical coefficients $a, b$ in (2) depend on liquid water content $W$ and the average droplets cloud radius $r_{0}$, constants are: $a \approx 1.45 W / r_{0}^{6}, b \approx 3 / r_{0}$.

Known model was developed earlier for small droplet assembles [10], it gave good description for special media like smoke or small industrial fogs where radii $\mathrm{r} \leq 1 \mu \mathrm{m}$. The Stokes friction for air flow at the droplet's surface provided particle motion inside acoustic wave, which is physical core of this model. For more large cloud droplets this model wrong, because is predicts the equality for the velocities of air $V_{a}$ and droplet $V_{w}$ for small frequencies $\omega$, see the first term in right side of result equation (4). The acoustic pressure is $P(t)=P_{a} \sin (\omega t), \omega$ is the circular frequency of the acoustic wave This model present equations with their solutions, as follows:

$$
\begin{gathered}
m \frac{d V_{w}}{d t}=6 \pi \eta r\left(V_{a}-V_{w}\right) \\
\tau \approx 0.22 \cdot \rho_{w} \cdot r^{2} / \eta \\
\tau \frac{d V_{w}}{d t}+V_{w}=V_{a, \max } \sin (\omega t) \\
V_{w}=\frac{V_{a, \max } \sin (\omega t-\varphi)}{\sqrt{1+\omega^{2} \tau^{2}}}+\frac{\omega \tau V_{a}}{1+\omega^{2} \tau^{2}} \cdot \exp \left(-\frac{t}{\tau}\right)
\end{gathered}
$$

There relaxation time is $\tau \sim 10^{-7} \mathrm{~s}$ for particles radius 0.1 $\mu \mathrm{m}$, but $\tau \sim 10^{-5} \mathrm{~s}$ for $r=1 \mu \mathrm{m}$, it provides zero in the second term in right side of (4) for low frequency then $t \sim \pi / \omega$. The medium/droplet delay is small here, $\varphi=\arctan (\omega \tau)$. This model was developed for small particles of smokes inside air flow when $r<<1 \mu \mathrm{m}$ was the norm. Therefore, using this model we found that the velocity amplitude of driving droplets of water becomes equal to the speed of the air molecules $V_{w} \approx V_{a}$, regardless of equation (4), when $\tau \omega<<1$ and $\omega$ up to $1 \mathrm{kHz}$. The calculations of (3-4) demonstrate the operation according to the model for a water droplets with only radii $r<1 \mu \mathrm{m}$. Acoustic wave increases non-linear motion with drift through the high power, such regimes are more preferable for small-sized industrial aerosol. Of cause, this is an incorrect approximation for large-sized droplets in clouds.

The new model is suggested here for large droplets inside clouds. Air molecules bombard great droplet surface to pass their impulse for droplet motion as a result with driving force amplitude $F$. It provide droplet vibrations back and forth in the acoustic wave; moves the droplets with an amplitude $L$ that has a maximal displacement during a time for half the period, as follows: 


$$
L_{\max }=\int_{0}^{\pi / \omega} V(t) d t
$$

there $V$ is the droplet amplitude. The modern complete model of one-dimensional vibration for a cloud droplet in a viscous medium can be found, for example, in [22-23], as follows:

$$
\begin{aligned}
& \left(\frac{4 \pi r^{3}}{3} \rho_{w}+f_{2}\right) y^{\prime \prime}+\left(f_{0}+f_{1}\right) y^{\prime}=F \cdot \sin (\omega t) \\
& f_{1}=6 \pi \eta_{a} r+3 \pi r^{2} \sqrt{2 \eta_{a} \rho_{a} \omega} \\
& f_{2}=3 \pi r^{2} \sqrt{2 \eta_{a} \rho_{a} / \omega}+\frac{2}{3} \pi \rho_{a} r^{3}
\end{aligned}
$$

where are water and air density are $\rho_{w}$, and $\rho_{a} ; \eta_{a}$ is medium viscosity, and $F$ is a driving force amplitude. The coefficients $f_{1}$ and $f_{2}$ correspond to the medium counteraction and are proportional to the speed and acceleration, respectively; $f_{0}$ corresponds to the inner mechanical losses that are, in reality, small. One can see that the Stoke's term, the first term of $f_{l}$ (7) is introduced into the motion equation (6). However, the second term in (7) provides the drag. The second part of $f_{2}$ characterizes the vibration of the joint mass of the medium surrounding a droplet, the first term in (8) corresponds to acoustic radiation losses. The solution of mentioned system (6-8) for the droplet velocity $V(t)=y^{\prime}$ provides the following equation:

$$
V=C \cdot \exp (-2 h t)+\frac{B}{(2 h)^{2}+\omega^{2}}[2 h \cdot \sin (\omega t)-\omega \cdot \cos (\omega t)]
$$

where normalized force is $B \approx F \backslash m \approx F \backslash\left(1.333 \pi r^{3} \rho_{w}\right)$. The attenuation parameter is $h$ which follows from the initial equation $V=0$ then $t=0$; also the integration-resulted term $C$ is as follows:

$$
\left.h=\frac{f_{0}+f_{1}}{f_{2}+4 \pi r^{3} \rho_{w} / 3}, \quad C=\frac{B \omega}{\omega^{2}+(2 h)^{2}}\right]
$$

The droplet displacement for half of the period of acoustic waves or vibrated droplet's amplitude is $L$ according to (5). The final solutions are as follows:

$$
\begin{aligned}
& V(t, \omega, h)=V= \\
& =\frac{B \omega}{(2 h)^{2}+\omega^{2}}\left[\exp (-2 h t)+\frac{2 h}{\omega} \cdot \sin (\omega t)-\cos (\omega t)\right] \\
& L=\frac{B}{\omega^{2}+(2 h)^{2}}\left\{2 \frac{2 h}{\omega}-\frac{\omega}{2 h}\left[\exp \left(-\frac{2 h \pi}{\omega}\right)-1\right]\right\}
\end{aligned}
$$

The driving force, $F$, should be determined at this point in order to obtain the numerical calculations, and it is necessary to set the predominant mechanism for the counteraction of acoustics using the weighted droplet in air to add the vibration. Here we assume the bombardment of the front surface of the large droplet by small air molecules; as well as the impulse transfer required to move the droplet to another location. The simplest formula of such a physical model describes the impulse transfer, $F \cdot \Delta t=\Delta p$. Side surface effects can be neglected for large droplet objects according to the next chapter consideration. The affecting force increases in proportion to the area of the front surface is $\sim r^{2}$. However, the droplet mass grows with radius as $\sim r^{3}$ that is mass driving is more important. Molecules bombard the droplet's surface during movement in an acoustic wave with a velocity of $V_{a}(t)=V_{a} \sin (\omega t)$. Molecule forward or back motions occur during the time of half period of the wave $t_{1 / 2}=\pi / \omega=$ $0.5 f^{l}$. The volume of air molecules in the front of the droplet is $U_{N}=S \cdot \int_{0}^{\pi / \omega} V_{a}(t) d t=\pi r^{2} V_{a} 2 / \omega$, but the cross-section of water droplet is $S=\pi r^{2}$. The complete mass for air molecules in this volume is $M_{a} \approx N \cdot 28 \cdot m_{p} \cdot U_{N}$. Here Avogadro's constant, and taking molecular mass for nitrogen is $2 \cdot 14 m_{p}$. We put to use for estimations the average molecule's velocity in acoustical wave $V_{a} / 2$. Driving force in acoustic wave according to second Newton's law could be found $F=$ $M_{a} \cdot \Delta V / \Delta t \sim M_{a} \cdot V_{a} / t_{1 / 2}$, and it corresponds to the next equation:

$$
F \approx\left(28 m_{p} N\right) \cdot r^{2} V_{a}^{2}=C_{F} \cdot r^{2} V_{a}^{2}
$$

where the coefficient is $C_{F} \approx 1$ in the SI system. The acoustic pressure, $P$, in the sound wave; the acoustic power, Q; the velocity, $V_{a}$, of the air molecules; their displacement, $L_{m}$; and the speed of sound $C_{a}$ are connected as follows:

$$
V_{a}=\sqrt{\frac{2 Q}{\rho_{a} C_{a}}}, P_{a}=\rho_{a} C_{a} V_{a}, L_{m}=\frac{1}{2 \pi f} \sqrt{\frac{2 Q}{\rho_{a} C_{a}}}
$$

Below we introduce the pulse power $J$, then $Q=J \cdot f$. After unification of (13-14) the driving force or large droplets in the acoustic wave is as follows:

$$
F \approx\left(\frac{2 C_{F}}{\rho_{a} C_{a}}\right) r^{2} J \cdot f, \mathrm{~B}=\mathrm{F} / \mathrm{mw}
$$

The droplet mass is $m_{w}=4 \pi r_{w}^{3} / 3$, so equations (12-15) for the droplet give the amplitude $L(f, r)$ in the from $L(f, r)$ :

$$
L(f, r)=\frac{J f}{r} \cdot \frac{C_{F 2}}{(2 h)^{2}+(2 \pi f)^{2}} \cdot\left\{\frac{2 h}{\pi f}+\frac{\pi f}{h} \cdot\left[1-\exp \left(\frac{-h}{f}\right)\right]\right\}
$$

There coefficient is $C_{F 2}=\frac{3 C_{F}}{2 \pi \rho_{w} C_{a} \rho_{a}}$.

Numerical calculations and the results are presented in Figure 2 according to this model (6-16). Figure 2 demonstrates examples for some optimized regimes for three acoustical ranges, as follows: (1) $f=20 \mathrm{~Hz}$ with lowest power $Q=175 \mathrm{~W} / \mathrm{m}^{2}$; (2) $f=50 \mathrm{~Hz}$ with $Q=800 \mathrm{~W} / \mathrm{m}^{2}$ and (3) $f=100 \mathrm{~Hz}$ with $Q=2500 \mathrm{~W} / \mathrm{m}^{2}$. Additional analysis were 
performed using the introduction of appropriate altitude changes relative to the main physical parameters in the model, as follows: air density $\left(\rho_{\mathrm{a}}\right)$, viscosity $(\eta)$, sound velocity $\left(C_{a}\right)$, and air temperature depending on km-altitudes. An improvement decrease in necessary acoustic power using the $2-6 \mathrm{~km}$ location of cloud with sound source within the atmosphere was observed. The calculations indicated a 10$15 \%$ increases for the amplitude values, $L$, according to the acoustics applied to the cloud drops directly at a altitudes of $6 \mathrm{~km}$ above the ground. For a typical cloud droplet, the estimations indicate that gravity is negligible small $(0.1 \%)$, as well as the drag force, due to the friction of air flow at the surface of the droplet. The irradiation time should be more longer for low frequency affects. We investigated the low frequency range from $f=50-100 \mathrm{~Hz}$ in order to provide an amplitude in a range $L \sim 0.5-2.5 \mathrm{~mm}$ for coalescence possibility. Calculations performed according to presented model indicated that the droplet amplitude becomes as high as $L \sim 2 \mathrm{~mm}$ for frequency $f=20 \mathrm{~Hz}$. Harmful frequencies are $7,14,21 \mathrm{~Hz}$; the first frequency is the body's heart pulse that should be not used.

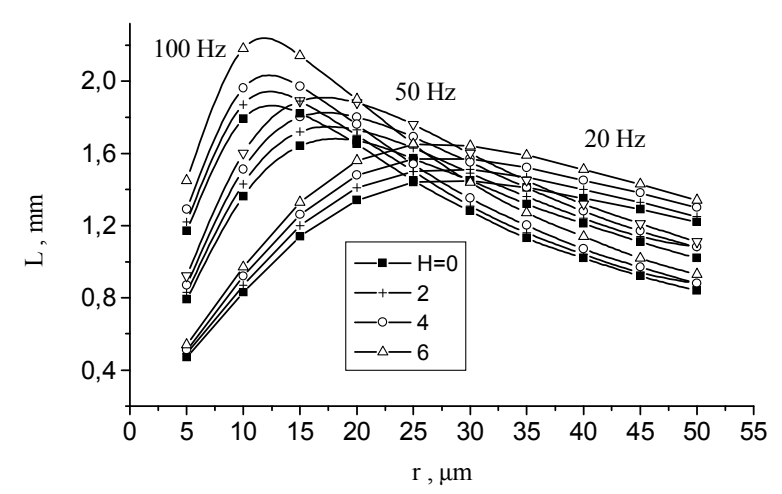

Figure 2. Vibration amplitudes $L$ for droplets radii 5 - $50 \mu \mathrm{m}$ for their location at altitudes $H=0,2,4,6 \mathrm{~km}$. The regimes are: $f=100 \mathrm{~Hz}$ and $Q$ $=2.5 \mathrm{~kW} / \mathrm{m}^{2} ; f=50 \mathrm{~Hz}$ and $Q=800 \mathrm{~W} / \mathrm{m}^{2} ; f=20 \mathrm{~Hz}$ and $Q=175 \mathrm{~W} / \mathrm{m}^{2}$.

Now we estimate the optimal average distance that should allow the droplets to collide. An average number concentration of droplets in a cloud occurs in the range from $N \sim 60-1000 \mathrm{~cm}^{-3}$ and more according to various measurements, see for example [12]. Here we assume that $N$ $\sim 500 \mathrm{~cm}^{-3}$ specifies the individual volume occupied by a weighted drop $1 / N$, and that its average distance is $L_{m} \sim N^{1 / 3}$ $\approx 1.26 \mathrm{~mm}$. The proposed regimes at Figure 2 provide necessary amplitudes for the typical ensemble of cloud droplets. The time requirement is $L_{m} / \mathrm{r} \approx 1260 / 10=2$ minutes or more to provide coalescence probability. The processes of coalescence/fragmentation for rainy droplets with mm-size range have been studied in a series of works both in theory and experiments [24]. The probability of the complete or partial amalgamation for two collision rainy droplets depends both on their sizes and velocities as listed below. Amalgamation for moving droplets with sizes ranges from $60<r_{s}<500 \mu \mathrm{m}, \sim 300 \mu \mathrm{m}$, and $0.5<r_{s}<1.5 \mathrm{~mm}$ for velocities, $V$, up to $8 \mathrm{~m} / \mathrm{s}$ have been investigated. Complete amalgamation for both colliding drops occurs then the parameter is in the range from $1<V^{2} \rho_{w} r_{s} / \sigma<4$ for small drops $\left(r_{s}\right)$ without high relative velocities $(V<0.4 \mathrm{~m} / \mathrm{s})$. Criterion for partial amalgamation was determined according to different experiments as the rage from $4<V^{2} \rho_{w} r_{s} / \sigma<50$, where $\sigma$ is a surface tension for a water.

\section{Optimal Acoustic Siren for Cloud Droplets}

Sirens are believed to be most suitable generators for powerful sound in air medium [25-26]. To produce a powerful nomo chromatic sound with a low-frequency we propose the air siren with special resonator that should be optimal for utilization in clouds. Monochromatic signal is more effective for droplet motion due to its flat wave front. Dynamic sirens are composed of a fixed stator with small holes in the periphery, and have a stator located inside of the rotating rotor with similar numbers of holes. The holes of the rotor and stator periodically overlap for the compressed air that comes out from siren core time to time. Outgoing air on the walls of the resonator sounds on own resonance frequency and power is sent to the outer media. The siren frequency is determined by the number of holes (or teeth) $z$, and the number of rotor circulation per minute, $n_{c}$, as follows $f=\left(z n_{c}\right) / 60$. For example, when $n_{c}=12.5 \mathrm{c}^{-1}$ and $z=8$, the frequency is $f=n_{c} \cdot z=100 \mathrm{~Hz}$. The frequency should be agreed with the rotor speed and own frequency of the resonator, so that maximum power output for the signal can be obtained. The siren utilized for our work was constructed and tested in experiments, so frequency change of the speed of rotation is accomplished using a connection by coupling the motor and the transformer with the variable voltage in order to speed tune for experiments.

Let's describe here the features of Bessel-form resonator to get pure monochromatic wave. The siren resonator serves for the concentration of acoustic energy with the selection of high spectrum into fundamental mode of resonator. Here we offer an optimal siren reflector based on the following analysis. The idea is that the shape of the optimal resonator for a siren should have a Bessel-formed wells in the reflector to maximize the power of acoustic radiation to a fundamental harmonic in a propagated wave within output beam. The fundamental harmonic distribution for energy in the radial direction is $J_{0}(k r)$ of propagated fundamental wave. The sound frequency is $f$; and the speed of sound $\mathrm{C}_{\mathrm{v}}$, for wavelength $(\Lambda)$, and the wave number $(k)$ are included as outlined below, when $f=100 \mathrm{~Hz}$.

$$
\mathrm{C}_{\mathrm{v}}=340 \mathrm{~m} / \mathrm{s}, \Lambda=\mathrm{C}_{\mathrm{v}} / \mathrm{f}=3.4 \mathrm{~m}, \mathrm{k}_{0}=2 \pi / \Lambda=2 \pi \mathrm{f} / \mathrm{C}_{\mathrm{v}}
$$

The first zero-solutions for the Bessel function is $x_{0}=2.404826$ for $J_{0}\left(k_{0} r_{0}\right)=J_{0}\left(x_{0}\right)=0$, such that the reflector's output radius, $r_{0}$, meters, can be modified to the formula $k_{0} r_{0} x_{0}=2.404826$, and as follows: 


$$
r_{0}(f)=\frac{x_{0}}{2 \pi \cdot f} \cdot C_{v}=\frac{130.13}{f}
$$

The calculated data for the outlet radiuses of Bessel's resonators are: $f=100 \mathrm{~Hz}$ needs $r_{0}=1.3 \mathrm{~m} ; f=150 \mathrm{~Hz}$ needs $r_{0}=0.868 \mathrm{~m}$ and etc. The complete mathematical representation for the series of resonator's waves follows to Bessel equation, and there is a proof for the optimal form of the sound reflector according to the Bessel wells. In another words, the shape of the reflector should be coincident with the contour of the zero-mode wave for the following reasons. Complete spectrum could be described in the sum of the converging and diverging waves of a cylinder waveguide, The spectrum of radiated harmonics with wavenumbers $k_{m, n}$ could be presented near no-optimized reflector outlet as follows:

$$
F(r, \varphi, t)=\sum_{m=0}^{\infty} \sum_{n=0}^{\infty} A_{m n} \cos (n \varphi) J_{m}\left(k_{m, n} r\right) \exp (i \omega t)
$$

We consider the centrally symmetrical modes without a series of waves with azimuthally variations, it gives the $n=0$ and $\cos (n \varphi)_{n=0}=1$. As discussed below, the boundary and initial equations have formulas:

$$
F(0, \varphi, t)_{r=0}<\infty, F\left(r_{0}, \varphi, t\right)_{r=r_{0}}=0,\left.F(r, \varphi, 0)\right|_{t=0}=f(r)
$$

The second boundary equation in (20) yields the zeromode Bessel function. All requirements of the boundary and initial equations provided the fundamental harmonic in radiated spectrum (19), as follows:

$$
F(r)=J_{0}\left(k_{0} r\right)
$$

Let's consider below the overlap of the side-well having Bessel form $f(r)=J_{0}\left(k_{0} r\right)$ with the radiated spectrum (19) to prove maximization of the power to fundamental harmonic by Bessel-form reflector. The function of the wells $f(r)$ could be presented in a series of Bessel functions anywhere, as follows:

$$
f(r)=A_{0} J_{0}\left(k_{0} r\right)+A_{1} J_{0}\left(k_{1} r\right)+\ldots,
$$

The unknown coefficients in (22) are determined as follows:

$$
A_{m}=\frac{2}{r_{0}^{2} J_{1}^{2}\left(k_{m} r_{0}\right)} \int_{0}^{r_{0}} r f(r) J_{0}\left(k_{m} r\right) d r
$$

Coefficients for harmonics $A_{0}$ and $A_{1}$ are:

$$
A_{0}=\frac{2}{r_{0}^{2} J_{1}^{2}\left(k_{0} r_{0}\right)} \int_{0}^{r_{0}} r J_{0}^{2}\left(k_{0} r\right) d r
$$

$$
A_{1}=\frac{2}{r_{0}^{2} J_{1}^{2}\left(k_{1} r_{0}\right)} \int_{0}^{r_{0}} r J_{1}\left(k_{0} r\right) J_{0}\left(k_{1} r\right) d r \ldots
$$

Bessel orthogonally functions gives:

$$
\begin{aligned}
& \int_{0}^{r} r J_{0}\left(k_{m} r\right) J_{0}\left(k_{m} r\right) d r=1 \quad, \quad m=n \\
& \int_{0}^{r} r J_{0}\left(k_{m} r\right) J_{0}\left(k_{n} r\right) d r=0 \quad, \quad m \neq n
\end{aligned}
$$

After unification the (26a) and (24) we get :

$$
\begin{aligned}
\int_{0}^{r 0} r J_{0}^{2}\left(k_{m} r\right) d r & =\frac{r_{0}^{2}}{2}\left[J_{0}^{\prime}\left(k_{m} r_{0}\right)\right]^{2}=\frac{1}{2} r_{0}^{2} J_{1}^{2}\left(r_{0} k_{m}\right) \\
& \Rightarrow \quad \mathrm{A} 0=1
\end{aligned}
$$

The higher-order harmonics of radiated spectrum should be equal to zero and $A_{m}=0$ according to (26b), (25). For comparison, let's consider usually used parabolic profile of the reflector wells to get pure spectrum, $f(r)=D-B \cdot r^{2}$, where $D$ and $B$ are the constants. Let's put this parabolic function to last of (20) and to equations (22-27) to found result radiation spectrum. The radiation waves for parabolic reflector will have higher harmonics, since $A_{0} \neq 1$ and $A_{m} \neq 0$ in (27) as follows:

$$
A_{0}=\frac{2}{r_{0}^{2} J_{1}^{2}\left(k_{0} r_{0}\right)} \int_{0}^{r_{0}} r\left[D-B r^{2}\right] J_{0}\left(k_{0} r\right) d r
$$

$$
\begin{aligned}
& \text { Here } D \int_{0}^{r_{0}} r J_{0}\left(k_{m} r\right) d r=D \frac{r_{0}}{k_{0}} J_{1}\left(k_{m} r_{0}\right) \text {, and } \\
& \qquad B \int_{0}^{r 0} r^{3} J_{0}\left(k_{0} r_{0}\right) d r=B \frac{r_{0}^{3}}{k_{0}^{2}} J_{1}\left(k_{0} r_{0}\right)-B \frac{2 r_{0}^{2}}{k_{0}} J_{2}\left(k_{0} r_{0}\right) .
\end{aligned}
$$

At the outlet of the reflector (at $x=2.4$ ), the edges should be folded outwards as a bell-shape to avoid edge diffraction with appropriate transfer of the radiated power into the higher harmonics. Technically, such a reflector could be manufactured using long and narrow strips of sheet of metal; strips could be assembled according to the principle of the fan and held together by transverse belts.

Let's calculate the output acoustic power of proposed siren. Various formulas were compared taken from well established approaches in [12, 26-29]. All of these author's formulas show yielded similar results for the calculations of the siren energy. Calculation model are based on propose that a siren is connected to any balloon where compressed air has a pressure of $P_{1}$, Bernoulli's equation indicates that for the velocity, $V$, of a gas stream through the holes area $S_{0}$ in rotor onto reflected walls at atmospheric pressure $P_{a}$. Various 
possible cases can be realized depending on the critical pressure $P_{0}=0.525 \cdot P_{1}$ (that is the pressure in the narrowest point of the out flowing jet), the connection is:

$$
\begin{aligned}
& P_{a}<P_{0}, V=\sqrt{\frac{2 \gamma}{\gamma-1} \frac{P_{1}}{\rho_{1}}} ; \\
& P_{a}>P_{0}, V=\sqrt{\frac{2 \gamma}{\gamma-1} \frac{P_{1}}{\rho_{1}}\left[1-\left(\frac{P_{a}}{P_{1}}\right)^{\frac{\gamma-1}{\gamma}}\right]} ; \\
& P_{1}>P_{0} /\left(\frac{2}{\gamma+1}\right)^{\frac{\gamma}{\gamma-1}} \approx 1.89 \cdot P_{0}, V=C
\end{aligned}
$$

There gas density in a balloon is $\rho_{1}$, and the heat capacities ratio is $\gamma=1.4$. Calculations performed according to the previous equations indicate, in particular, that the pressures in a cylinder $2-3 \mathrm{~atm}$ produces gas outflow with velocity $\mathrm{V}$ $\approx 200-300 \mathrm{~m} / \mathrm{c}$. A further increase in the pressure is impractical, because for the same time interval the velocity of the gas grows very slowly. One way to determine estimations using the necessary acoustic energy is [29], as follows:

$$
Q_{0} \approx \eta \alpha(1+\delta) S_{0} P_{a}\left(2 g R T_{1}\right)^{1 / 2}\left(\frac{\gamma}{\gamma-1}\right)^{\frac{3}{2}} \frac{P_{a}}{P_{1}}\left[1-\frac{P_{0}}{P_{1}}\right]^{\frac{\gamma-1}{\gamma}}
$$

where $T_{1}$ is the gas temperature in a balloon, $R$ is the universal gas constant, and $\gamma=1.4$, as an example of an output acoustic power calculation. This siren can have 8 holes with radii equal to $3.5 \mathrm{~mm}$, and their summarized surface is $S_{0}$. Coefficients $\varepsilon \sim 0.5$ for the energy characteristics that overlap in siren teeth during rotation, and for which the $\delta$ coefficient also indicates the proportion of air flow between the stator and the rotor, generally lead to $\alpha_{c}(1+\delta)=0.7-1.4 \sim 1$. If there is a good efficiency $\eta_{a} \sim 0.3$, and if air pressure in the balloon is $P_{1}=2 \mathrm{~atm}$, the air output flow gives $\sim 0.1 \mathrm{~m}^{3} / \mathrm{s}$, and the output acoustic power is $Q_{0} \approx$ $2.24 \mathrm{~kW}$. Calculations for the ratio of the output power, $Q_{0}$, to the square of the output area $\pi r_{0}^{2}$ of the siren reflector are presented in Table 2. When the power $Q_{0}=3.89 \mathrm{~kW}$ was received from the siren then the pressure in the balloon increased up to $P_{1}=3 \mathrm{~atm}$. Results calculations are presented at Table 2, there siren power was divided for output resonator square $\pi r_{0}^{2}$, but resonator output radius $r_{0}$ was calculated from equation (18).

The comparison of the siren power at Table 2 with necessary data at Fig. 2 predict that optimal regimes are $f=$ $100-200 \mathrm{~Hz}$ with output siren power $Q_{0} \approx 4 \mathrm{~kW}$. The evaluation indicated that require acoustic power from the siren was not very high for low-frequency regimes, but vibration algorithm to be valid here principally. The siren will be more effective then the average number concentration of droplets in a cloud $N$ is higher then considered here number $\left(500 \mathrm{~cm}^{-3}\right)$, let's demand $N>500$. Another simplest possibility is the number tithes increase with siren power increase proportionally.
Table 2. Acoustic power $Q_{0}\left(\mathrm{~W} / \mathrm{cm}^{2}\right)$, when the pressure inside siren is $P_{1}=$ 2 - $3 \mathrm{~atm}$.

\begin{tabular}{lll}
\hline $\boldsymbol{f}, \mathbf{H z}$ & $\begin{array}{l}\boldsymbol{Q}_{0} / \boldsymbol{\pi}_{0}{ }^{2}, \mathrm{~W} / \mathbf{m}^{2} \\
\left.\text { (then } \boldsymbol{P}_{\boldsymbol{1}}=\mathbf{2} \mathbf{a t m}\right)\end{array}$ & $\begin{array}{l}\boldsymbol{Q}_{0} / \boldsymbol{\pi}_{0}{ }^{2}, \mathrm{~W} / \mathbf{m}^{2} \\
\left.\text { (then } \boldsymbol{P}_{\boldsymbol{1}}=\mathbf{3} \mathbf{a t m}\right)\end{array}$ \\
\hline 20 & 17 & 29 \\
50 & 106 & 183 \\
100 & 423 & 734 \\
200 & 1690 & 2932 \\
\hline
\end{tabular}

\section{Conclusion}

The calculations correspond to the regimes of acoustic power that are required for implementing the vibration model of cloud droplets according to proposed new low-frequency model. The siren construction is recommended with enough output power. By taking the above into account we concluded that the proposed acoustic method is up to date and does not require extensive economic support. Additionally, artificial rains have desirable features for air purification and climate corrections. Acoustic method provide fast droplets coalescence inside nature clouds and sedimentation "to get rain at the right time".

\section{References}

[1] Langmuir, I. and V.J. Schaefer, 1937: Improved methods of conditioning surfaces for adsorption. J. Am. Chem. Soc., 59, $1762-1763$

[2] Arnett S Dennis, 1980: Weather Modification by Cloud Seeding. Academic Press Inst, New York. 267p.

[3] Mather, G.K., and D.F. Terblanche, 1997: Results of South African cloud - seeding experiments using hygroscopic flares. J. Appl. Meteorology, 36, 1433-1447.

[4] Rauber, R.M., Steven, J.: Rain in shallow cumulus over the ocean. BAMS, 88, 1912-1928, 2007.

[5] Shmeter, S.M., and Beryulev, G.P.: Efficiency of cloud and precipitation modification with hygroscopic aerosols. Rus. Meteorology and Hydrology, 2, 43-60, 2005.

[6] Drofa, A.S. et al., 2006: Formation of cloud microstructure: the role of hygroscopic particles. Izvestiya. Atmospheric and oceanic physics, 42, 355-366.

[7] Lau, K.-M., Ramanathan V., et all., 2008: The joint aerosolmonsoon experiment. A new challenge for monsoon climate research. BAMS, 89, 369-383.

[8] Tulaikova, T.V., Micjetchenko A.V., Amirova S.R.,: Acoustic rains (Physmathbook, Moscow, 2010).

[9] Tulaikova, T., and Amirova, S.: The effective possibility for atmosphere CO2 purification (Lap-Lambert, Germany, 2012

[10] Fuchs, N.A.: The Mechanics of Aerosols. Pergamon, New York, 1964.

[11] Boucher, R.M.G., 1960: Acoustical energy in fog dispersal techniques. Ultrasonic news, 4, 11-19.

[12] Mednikov, A.P., 1965: Acoustic coagulation and precipitation of aerosols. New York, Consultants Bureau. 
[13] Gorbachev, L.P., A.B. Severniy, 1936: Additions to the effects of sound waves on the droplets of mist. Journal of Technical Physics, 7, 536-545

[14] IPCC, 2014: Summary for Policymakers, In: "Climate Change 2014, Mitigation of Climate Change. Contribution of Working Group III to the Fifth Assessment Report of the Intergovernmental Panel on Climate Change”, Edenhofer, O., and all. (Cambridge University Press, Cambridge, United Kingdom and New York, NY, USA, 2014).

[15] Kleidon, A., Malhi, Y. and Cox, P.M.: Maximum entropy production in environmental and ecological systems introduction. Philos. T R Soc. B, 365, 1297-1302, 2010.

[16] Shepherd, J.,: Geoengineering the climate: science, governance and uncertainty (The Royal Society, London, 2009).

[17] Corner K. A. Absorption of sound waves with finite amplitude. Acoustic journal (Rus), 1958, vol.2, 115-124.

[18] Goldberg H. A. On the propagation of plane waves with finite amplitude. Acoustic journal (Rus), 1957, vol.4, 322-327.

[19] Ostashev C. E. Sound propagation in moving media. M.: Nauka, 1992.

[20] Connolly P.J., Sauders C.P.R., Gallagher M.W., Bower K.N., Flynn M.J., Choularton T.W., Whiteway J.A., Lawson R.P. Aircraft observation of the influence of electric fields on the aggregation of ice crystals. // Q.J.R. Soc., 2005, 131, p. 16951712 .
[21] Connolly P.J., Choularton T.W., Gallagher M.W., Bower K.N., Flynn M.J., Whiteway J.A.. Cloud -resolving simulations of intense tropical Hector thunderstorms implications for aerosol-cloud interaction // Q.J.R. Met. Soc, 2006, 132, p. 3079-3106.

[22] Prokhorov A.M., Claus R.O., Popov A.M., Tulaikova T.V. Modeling of the fiber-optical sensor based on micromechanical vibrations J.Appl.Opt,36,1997, p.5562-5565.

[23] H. Kumazaki, S. Inaba, K. Hane. Temperature characteristics of vibrating type sensor using micromachined optical fiber-tip. Optical review, 1999, vol.3, p.135-138.

[24] Mason B. J. Physics of clouds. Oxford, Clarendon Press, 1971.

[25] Hartman J. The acoustic air-jet generator // Ingenirovidenskabelige skrifter, 4, 1, 1939.

[26] Gladyshev V. N. Dynamic siren. Theory. Experiment. Novosibirsk.: Geo Publishing, 2000.

[27] Karnovsky, M.I., 1945: Theory and calculations for siren. Journal of technical physics, XV, 348-144.

[28] Shkundin, S.Z., O.A. Kremleva, V.A. Rumantsev, 2001: The theory of acoustic anemometry. Moscow, Russia.

[29] Podoshevnikov, B.F., V.A. Grebemchuk, V.P. Kurkin, 1961: Acoustic coagulation of aerosols. GNTI, Moscow. 